\title{
BigDataProcessor2: a free and open-source Fiji plugin for inspection and processing of TB sized image data
}

\author{
Christian Tischer ${ }^{1,2,3, *}$, Ashis Ravindran ${ }^{4}$, Sabine Reither ${ }^{2,3}$, Nicolas Chiaruttini ${ }^{5}$, \\ Rainer Pepperkok ${ }^{2,3}$ and Nils Norlin (10 ${ }^{3,6,7, *}$
}

${ }^{1}$ Centre for Bioimage Analysis, European Molecular Biology Laboratory (EMBL), 69117, Heidelberg, Germany, ${ }^{2}$ Advanced Light Microscopy Facility, EMBL, 69117, Heidelberg, Germany, ${ }^{3}$ Cell Biology and Biophysics Unit, EMBL, 69117, Heidelberg, Germany, ${ }^{4}$ University of Heidelberg, Department of Scientific Computing, 69120, Heidelberg, Germany, ${ }^{5}$ Biolmaging \& Optics Platform (BIOP), Faculty of Life Sciences (SV), Ecole Polytechnique Fédérale de Lausanne, CH-1015 Lausanne, Switzerland, ${ }^{6}$ Department of Experimental Medical Science, 221 84, Lund University, Lund, Sweden and ${ }^{7}$ Lund University Bioimaging Centre, 222 41, Lund University, Lund, Sweden

*To whom correspondence should be addressed.

Associate Editor: Jinbo Xu

Received on August 12, 2020; revised on February 2, 2021; editorial decision on February 6, 2021; accepted on February 13, 2021

\begin{abstract}
Summary: Modern bioimaging and related areas such as sensor technology have undergone tremendous development over the last few years. As a result, contemporary imaging techniques, particularly electron microscopy (EM) and light sheet microscopy, can frequently generate datasets attaining sizes of several terabytes (TB). As a consequence, even seemingly simple data operations such as cropping, chromatic- and drift-corrections and even visualisation, poses challenges when applied to thousands of time points or tiles. To address this we developed BigDataProcessor2-a Fiji plugin facilitating processing workflows for TB sized image datasets.

Availability and implementation: BigDataProcessor2 is available as a Fiji plugin via the BigDataProcessor update site. The application is implemented in Java and the code is publicly available on GitHub (https://github.com/bigda taprocessor/bigdataprocessor2).
\end{abstract}

Contact: christian.tischer@embl.de or nils.norlin@med.lu.se

Supplementary information: Supplementary data are available at Bioinformatics online.

\section{Introduction}

Inspection and processing of TB sized image data as produced by state-of-the-art light-sheet and volume electron microscopy poses several practical challenges (Power and Huisken, 2017). Even image inspection can be burdensome, because loading the entire dataset from disk into Random Access Memory (RAM), as it is usually done for conventional MB to GB sized data, is not feasible due to the limitations of a standard computer's RAM. In addition, pixel-wise image processing operations on the whole dataset can take hours and may require data duplication on disk as the processed images cannot be held in RAM.

The challenges of big image data inspection can be addressed by lazy-loading schemes where only the portion of the data is loaded into RAM that is needed to render the current view on the computer monitor. There are several commercial and open source solutions that adopt lazy-loading to interactively render big image data. The rendering modes include fixed angle 2D slicing: ImageJ (Schneider et al., 2012); arbitrary angle 2D slicing: BigDataViewer (Pietzsch et al., 2015); and 3D volume rendering: Imaris (Oxford Instruments), Arivis Vision4D (Arivis AG), Amira (Thermo Fisher Scientific), Vaa3D (Bria et al., 2016) and TDat (Li et al., 2017). However, except for the fixed angle 2D slicing mode, these solutions require the data to be saved in specifically chunked multiresolution file formats that enable efficient loading of arbitrary data portions from disk into RAM. Due to write performance considerations, raw microscopy data is typically not saved in a format that is compatible with those requirements. In addition, raw microscopy data can have further shortcomings. For example, only parts of the acquired data may be of actual interest, either because larger fields of view have been acquired to compensate for unpredictable sample motion, or scientifically interesting phenomena have only occurred in specific parts of the imaged sample. Also, pixel density and bit-depth can be unnecessarily high, because camera-based microscope systems with fixed pixel size and bit-depth have been used. Moreover, there may be chromatic aberrations or other shifts between acquired data channels or time points.

Taken together, to render raw microscopy data amenable for analysis it typically needs to be re-saved in a suitable file format and a 
(a)

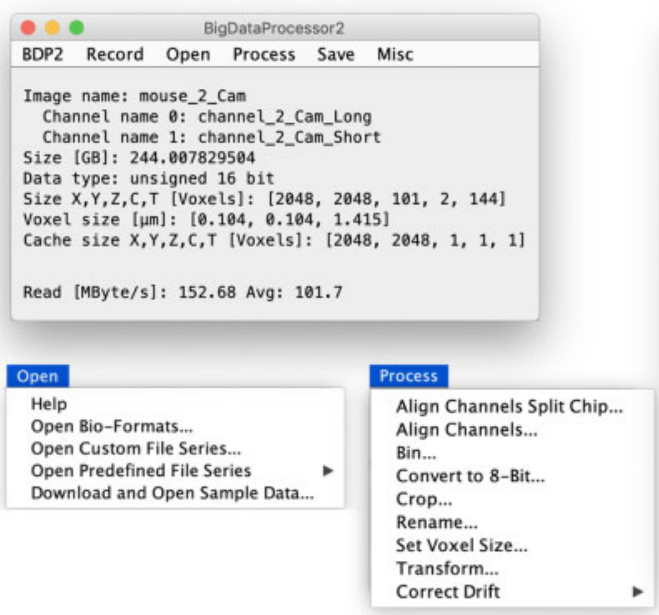

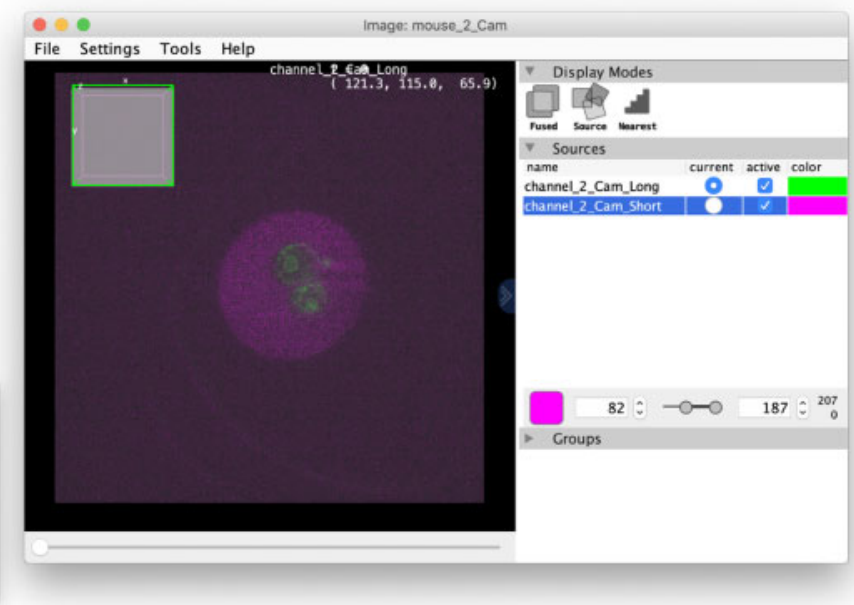
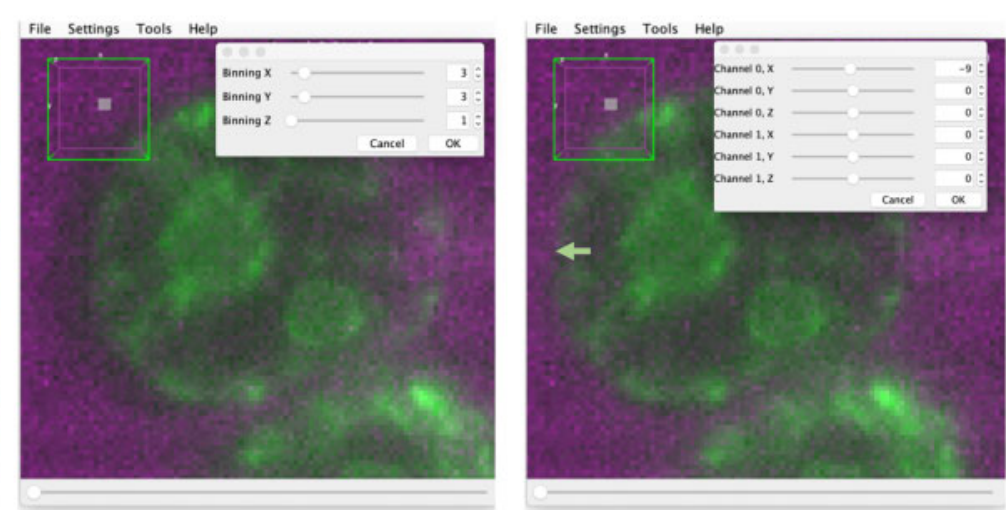

(b)

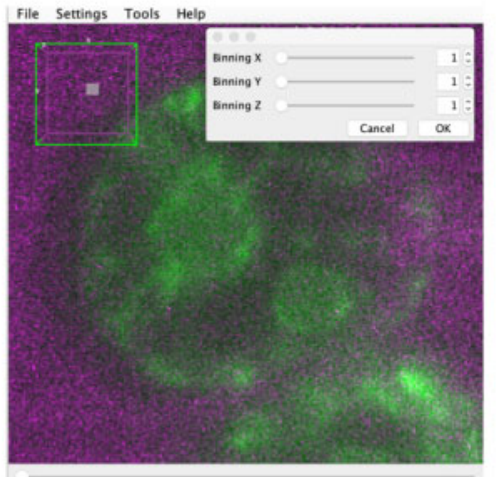

(c)
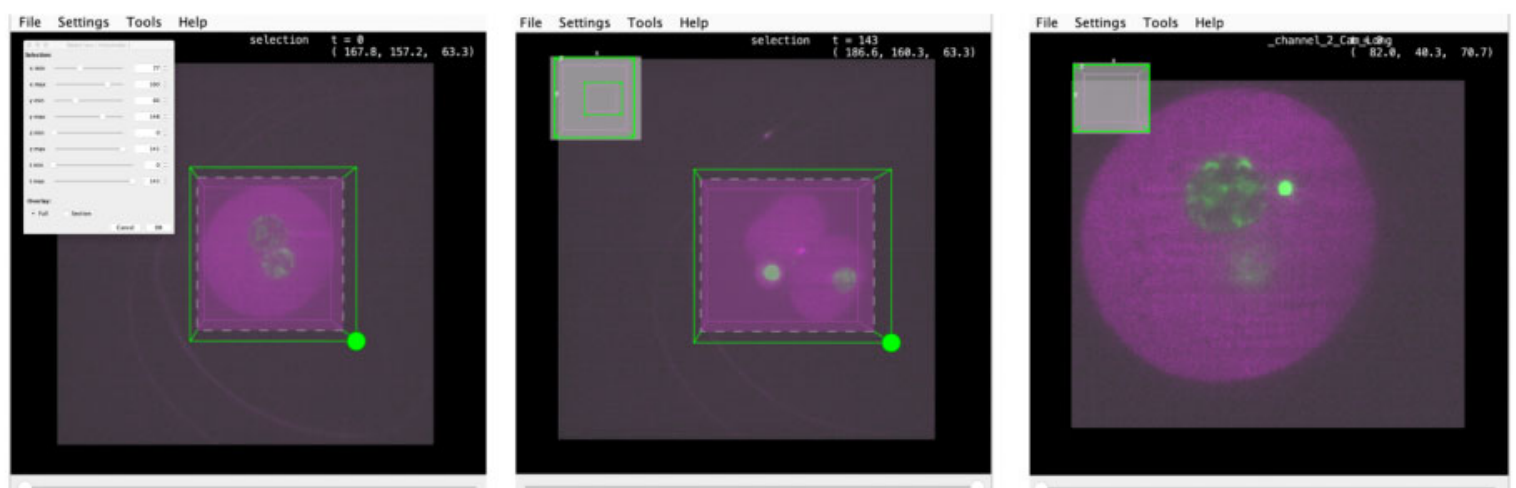

Fig. 1. Overview of the user interface and an example workflow in BDP2. (a) Image browsing. Left: The main BDP2 window with the Open and Process dropdown menus expanded (see also Supplementary Note S1). Right: BigDataViewer's user interface. (b) Binning and channel alignment. Left: Original data (zoomed in). Middle: $3 \times 3$ binning in $X \& Y$, reducing noise and the size of the dataset by a factor of $3 \times 3=9$. Binning (and all other processing operations) is performed by means of lazy computation and can thus be configured interactively even for TB sized datasets. Right: Channel alignment, correcting a shift (green arrow) of the green relative to the magenta channel. (c) Cropping. Left: First time point of the dataset with interactive cropping user interface. Middle: Last time point of the dataset with enlarged cropping area to include all relevant data. Right: Cropped data, showing again the first time point. Thanks to BDP2's lazy-loading and lazy-processing the above steps (a-c) can be executed in a few minutes. Finally, the data could be re-saved using the Save menu (not shown). In this example, binning and cropping helped to reduce the size of the data from 244 to 4.8 GB without loss of biologically relevant information

number of processing operations such as cropping, binning, bit-depth conversion, channel shift and drift correction might need to be applied. It is important to realize that, if performed sequentially, each of these processing steps requires loading, processing and re-saving of the entire initially TB sized dataset, which, taken together, could take several hours or even days. It would be much more efficient to load the raw data, apply all processing steps and then re-save the data only once.
To this end, making use of ImageJ's virtual stacks (Schneider et al., 2012) for lazy-loading of big image data we previously developed BigDataProcessor1 (https://github.com/bigdataprocessor/bigda taprocessor1), a Fiji plugin (Schindelin et al., 2012) facilitating interactive browsing, processing and re-saving of TB sized microscopy raw data. BigDataProcessor1 has proven useful to conduct big image data processing workflows similar to the one shown in Figure 1 (e.g. 
Alladin et al., 2020, Villani et al., 2019, Wolny et al., 2020). We thus decided to reimplement and enhance BigDataProcessor1 using software libraries dedicated to lazy-loading and lazy-processing of big image data (Supplementary Note S9). The resulting BigDataProcessor2 is described in this application note.

\section{Implementation and application}

BigDataProcessor2 (BDP2, https://github.com/bigdataprocessor/big dataprocessor2) is built on ImgLib2 (Pietzsch et al., 2012), a Java library for processing of $\mathrm{n}$-dimensional big image data. BDP2 can be installed by activating the 'BigDataProcessor' Fiji update site. All functionality is accessible both via a graphical user interface (Fig. 1a) and via recordable scripts (Supplementary Note S2). BDP2 supports loading of $5 \mathrm{D}(x, y, z$, channel and time) image data via Bio-Formats (Linkert et al., 2010) as well as loading TIFF (https://www.adobe.io/open/stand ards/TIFF.html) and some HDF5 (The HDF Group, 1997) based image files series (Supplementary Note S3), thereby accommodating a majority of image data formats currently occurring in light sheet and volume electron microscopy. For visualisation, BDP2 employs BigDataViewer (Pietzsch et al., 2015), which provides arbitrary plane slicing of $5 \mathrm{D}$ image data. As one microscopy camera image typically contains only a few MB of data, it fits readily into RAM and can be loaded within sub-seconds, given a data access bandwidth larger than or equal to a few MB/s. These bandwidths are nowadays typically available (Supplementary Note S7) and as such BDP2's lazy-loading scheme allows for interactive browsing of TB sized image data on a standard computer (Supplementary Note S8).

Importantly, BDP2 also provides lazy image processing operations reducing computations to only the pixels needed to render the currently viewed image plane (Supplementary Fig. S1 and Supplementary Note S9). At present, we support the following lazy processing operations (Supplementary Note S4): affine transformed viewing, cropping, binning, bit-depth conversion, drift correction and channel alignment (chromatic shift correction, split chip acquisition). Additional processing operations can be contributed via the SciJava plugin framework (see https:/github.com/bigdataprocessor/bigdataprocessor2/blob/mas ter/CONTRIBUTE.md). The compute times of currently available operations are typically in the sub-second range for one image plane (Supplementary Note S7) such that the user can interactively configure all image processing steps while inspecting arbitrary locations in the sample (Fig. 1 and Supplementary Movies S1 and S2). Once all processing steps have been configured, the dataset can be re-saved for further analysis. We currently provide saving in TIFF and HDF5 based formats, including lossless compression, with one file per 3D volume (Supplementary Note S5). For TIFF, we also support saving each plane to a separate file. For HDF5, we save the data in a chunked pyramidal format allowing for efficient viewing with both BigDataViewer and Imaris (Imaris v9.0, Bitplane AG).

\section{Discussion and conclusions}

Visualisation and processing of an image dataset conventionally comprises loading this dataset from a storage device such as a hard disk into a computer's RAM from where it is accessed and processed by the computer's CPU. With the increasing size of image data in biological research this mode of operation is becoming challenged.

Due to the size and to ensure data integrity, datasets are typically not stored on the processing computer itself but inside the research institute's data centre, where it is accessed via a local area network (LAN) connection. Loading a medium sized light sheet dataset (16 bit, $2048 \times 2048 \times 100 \times 2 \times 100 ; x, y, z$, channels, time points) via a $1 \mathrm{Gbit} / \mathrm{s}$ LAN connection would take $22 \mathrm{~min}$ and require $168 \mathrm{~GB}$ of RAM. Applying a typical processing operation such as a $3 \times 3 \times 1(x, y, z)$ average binning using ImageJ's Transform $>$ Bin command on a $2.5 \mathrm{GHz}$ Intel Core i7 (i7-7660U) on the whole dataset would take about $158 \mathrm{~s}$ (about 0.5 Giga voxels/s). This example shows that processing operations on whole big image datasets require high-end computer hardware with a large RAM and parameters of processing operations cannot be interactively configured as they take more than a couple of seconds to complete. Even if there were hardware solutions to achieve userfriendly real-time performance we argue it is more compute resource efficient to only process and inspect a representative subset of the data to judge whether a processing step is adequately configured.

Once an appropriate processing pipeline is configured, the whole dataset can be efficiently processed and re-saved in one go. Notably, the processed data can be significantly smaller than the raw data (Fig. 1) and thus much more efficient to work with. Unfortunately, no standardized big image data file format has yet emerged and thus one may have to re-save the data multiple times in formats optimized for specific image analysis and visualization software. Thus, we consider the establishment of a standard big image data file format as a very important endeavour for the bioimaging community within the next few years (Swedlow et al., 2020).

In conclusion, we consider our BigDataProcessor2 Fiji plugin to substantially simplify the inspection and processing of big image data. As big image data is becoming increasingly prevalent we are positive that the already existing user base will grow even further in the future.

\section{Acknowledgements}

The authors thank the EMBL IT-department for support, Tobias Pietzsch (MPI CBG, Dresden) for help related to BigDataViewer, AshnaAlladin, Matthew Boucher, Isabell Schneider (EMBL Heidelberg), Gustavo QuintasGlasner de Medeiros (FMI Basel), Alexis Maizel (University Heidelberg), Marion Louveaux (Institut Pasteur), Jan Rhoden (Bruker), BjoernEismann (Bruker), the Francesca Peri lab (University of Zürich), Robert Haase (PoL TU Dresden) and our second reviewer for testing, reading the manuscript and/or providing suggestions.

\section{Funding}

This work was supported by the Åke Wiberg foundation, Per-Eric and Ulla Schyberg's foundation, IngaBritt and Arne Lundberg foundation, Royal Physiographic Society of Lund, Sigurd and Elsa Goljes Memorial Foundation and a Corbel II grant (N.N.).

Conflict of Interest: none declared.

\section{References}

Alladin, A. et al. (2020) Tracking cells in epithelial acini by light sheet microscopy reveals proximity effects in breast cancer initiation. eLife, 2020, 9: e54066.

Bria, A. et al. (2016) TeraFly: real-time three-dimensional visualization and annotation of terabytes of multidimensional volumetric images. Nat. Methods, 13, 192-194.

Imaris v9.0 Bitplane AG.

Li, Y. et al. (2017) TDat: an efficient platform for processing petabyte-scale whole-brain volumetric images. Front. Neural Circuits, 11, 51.

Linkert, M. et al. (2010) Metadata matters: access to image data in the real world. J. Cell Biol. 189, 777-782.

Pietzsch, T. et al. (2015) BigDataViewer: visualization and processing for large image data sets. Nat. Methods, 12, 481-483.

Pietzsch, T. et al. (2012) ImgLib2 - generic image processing in Java. Bioinformatics, 28, 3009-3011.

Power, R.M. and Huisken,J. (2017) A guide to light-sheet fluorescence microscopy for multiscale imaging. Nat. Methods, 14, 360-373.

Schindelin, J. et al. (2012) Fiji: an open-source platform for biological-image analysis. Nat. Methods, 9, 676-682.

Schneider, C. et al. (2012) NIH Image to ImageJ: 25 years of image analysis. Nat. Methods, 9, 671-675.

Swedlow, J. et al. (2020) A Global View of Standards for Open Image Data Formats and Repositories. Arxiv:2010.10107v1.

The HDF Group.(1997) The HDF Group. Hierarchical Data Format, version 5, 1997-NNNN.

Villani, A. et al. (2019) Neuronal clearance by microglia depends on packaging of phagosomes into a unique cellular compartment. Dev. Cell, 49, 77-88.

Wolny, A. et al. (2020) Accurate and versatile 3D segmentation of plant tissues at cellular resolution. eLife, 2020, 9:e57613. 